\title{
Identifying Artifacts from Real Changes in Electron Microscopy of Molecular Era
}

\author{
Gang Ning ${ }^{1}$
}

1. Microscopy Facility, Huck Institutes of the Life Sciences, Penn State University, University Park, PA, USA.

The recent advancements of molecular biology enable us to study mechanisms underlying how a specific gene governs correspondent specific functions, and the study of these functions are usually related to investigation of phenotypes of experimental models that we used in these studies. Microscopy, especially electron microscopy, is a frequently used methodology to investigate the changes, in many cases very subtle ones, in genetically altered mutants of animal, plant, insect, and microbe models. There are also new probes and tracers, such as quantum dots and nanoparticles, used in the imaging research. In practice, it is found that there are many occasions where artifacts can be mistaken as real changes and vice versa. Therefore, it is crucial to identify artifacts from real changes caused by genetic alterations in nowadays' electron microscopy.

It is well a known phenomenon that glutaraldehyde, an aldehyde fixative for conventional TEM primary fixation, can cause plasma membrane blistering of cells during fixation [1]. We have observed remarkable membrane blistering in virus-infected cultured cells upon addition of glutaraldehyde fixative. This artifact can be confused with cellular damages caused by viral infection. Cryo-fixation by plunge or high-pressure freezing, where cells are instantly vitrified, has been used to avoid this artifact. In other hand, it can be challenging that the cryo-fixation is restricted by equipment requirement and complicated sample handling. As a result, limited number of samples and relatively small quantity of cells are available for statistical analysis in cryo samples. For example, the separation of cell envelope (including capsule, inner and outer membrane), an artifact caused by freezing membrane cracks, are often detected in cryo-fixed bacteria, especially in gram-negative bacteria, which can be confused with real changes in mutant cell envelope. We found conventionally chemical-fixed samples that were processed parallel with cryo-fixed ones could give additional evidence to support the mutant changes and thus provide convincing data due to their quantity of sample for statistical analysis (Fig. 1).

Control group is of essential importance in the microscopic studies. In a SEM study of malaria $P$. falciparum infected human RBCs, star-shaped cells were observed in mutant and in wild type parasites as well (Fig. 2). Results of statistical analysis suggested there were likely significant differences of this type of changes between mutant and wild type. It had not been found out until later time, however, these starshaped cells were actually some unhealthy RBCs in the sample, when an uninfected RBC sample was processed and imaged to rule out it was a change caused by genetic alteration of the mutant. Therefore, uninfected control samples must be included in the study of infection of pathogen mutant.

With computer-aided digital imaging and fully motorized sample stages, large data sets can be obtained easier than ever for performing statistical analysis by using montage, multi-point imaging, block-face imaging SEM as a few examples. Correlative light and electron microscopy (CLEM) is a preferable method to avoid artifacts. Correct use of control group, experience in sample preparation, and experience to be able to identify artifacts are still among the basic skills to lead correct scientific conclusions in the electron microscopy of molecular era [2]. 
References:

[1] Bowers B in "Artifacts in Biological Electron Microscopy", ed. Crang R. F. and Klomparens K. L., (Plenum Press, New York) p. 13.

[2] Author thanks Huck Institutes of the Life Sciences at Penn State University Park for supporting this study.
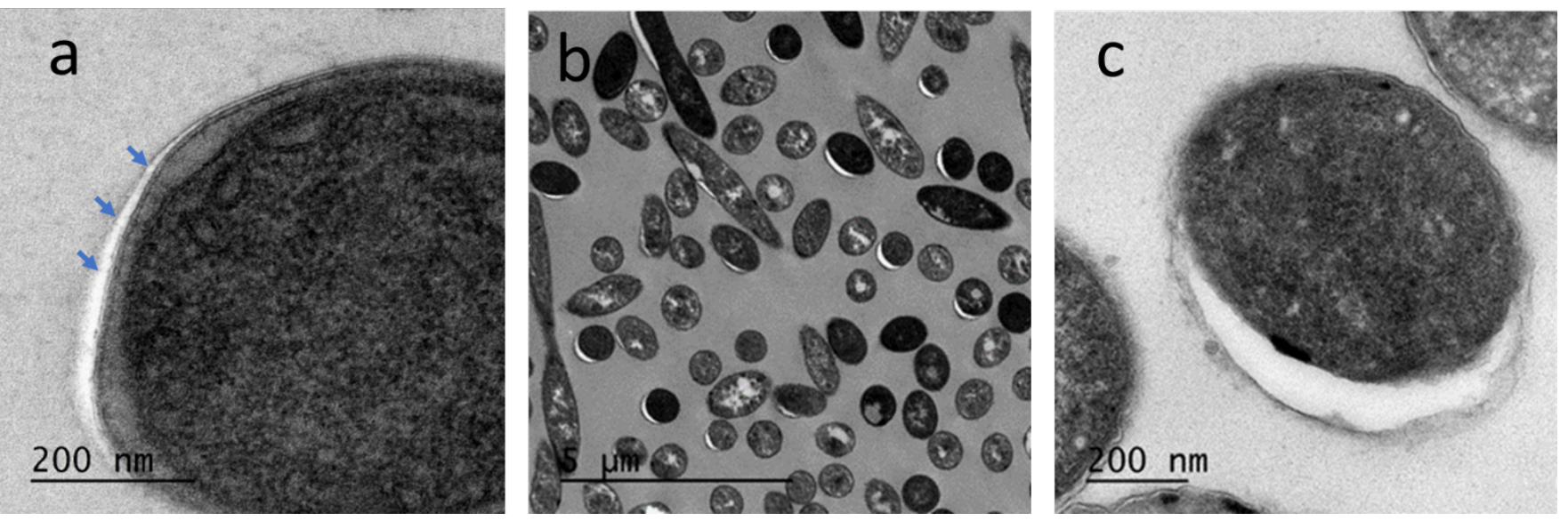

Figure 1. Membrane separation of bacterial envelope in cryo-fxied samples of mutant strain (arrows in a) is difficult to rule out it is an artifact derived from freezing process whereas conventional EM (b, c) provides additional evidence supporting the change is real by a large number of cells with separation, but is not able to show clear membranal continuation because of wrinkled membrane.
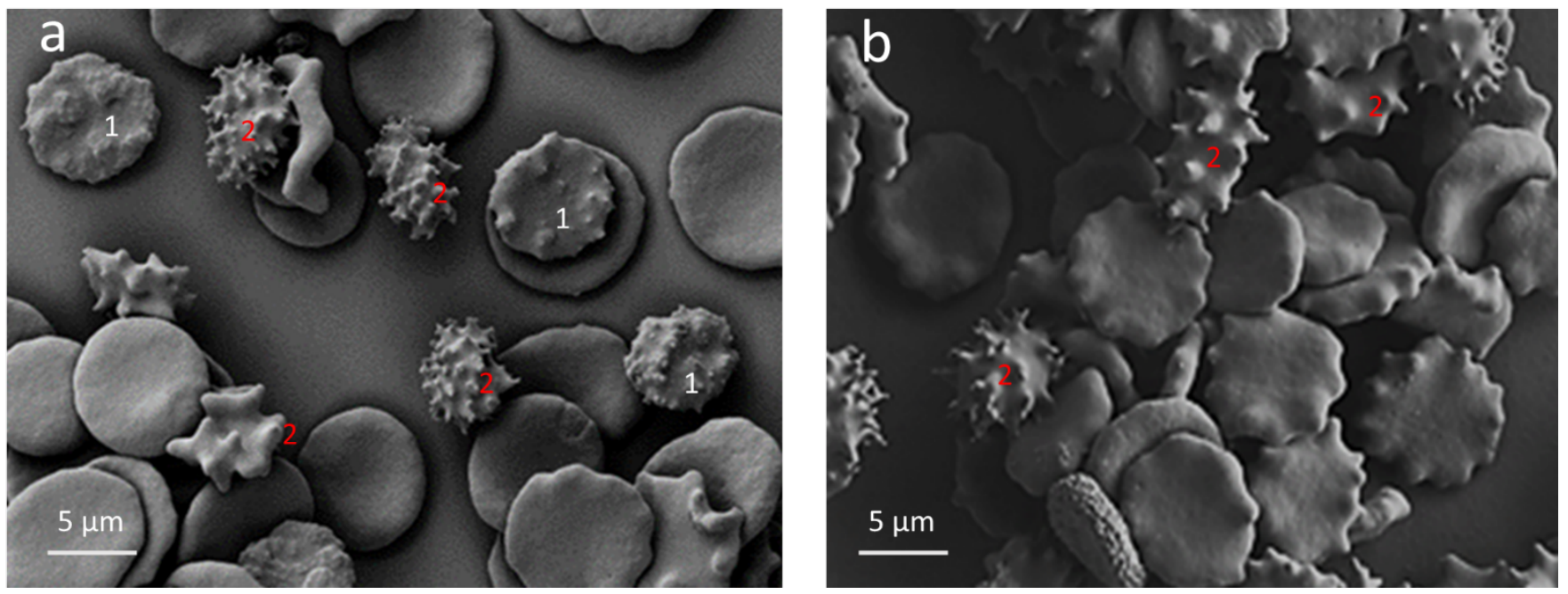

Figure 2. Human RBCs infected by a mutant malaria $P$. falciparum (a) appeared to have two types of changes ( 1 and 2 ) but only Type 1 is a real change caused by the infection. Type 2 can also be identified in uninfected RBCs (b) as unhealthy cells in the sample. 